Institute for Immunology and Informatics and Department of Cell and Molecular Biology. University of Rhode Island, Providence, Rhode Island 02903, USA.

e-mail:

arothman.uri@gmail.com doi:10.1038/nri3014

Published online 15 July 2011

\title{
Immunity to dengue virus: a tale of original antigenic sin and tropical cytokine storms
}

Alan L. Rothman

Abstract | Dengue is a mosquito-borne viral disease of expanding geographical range and incidence. The existence of four viral serotypes and the association of prior dengue virus infection with an increased risk for more severe disease have presented significant obstacles to vaccine development. An increased understanding of the adaptive immune response to natural dengue virus infection and candidate dengue vaccines has helped to define the specific antibody and $\mathrm{T}$ cell responses that are associated with either protective or pathological immunity during dengue infection. Further characterization of immunological correlates of disease outcome and the validation of these findings in vaccine trials will be invaluable for developing effective dengue vaccines.

Dengue as a clinical entity is thought to date back at least several hundred years, and its aetiology was recognized as a mosquito-borne virus nearly 100 years ago. Military physicians in the United States and Japan began efforts to develop a vaccine against dengue during the Second World War, when the virus strains were isolated. Progress since that time has been disappointingly slow. The geographical range of dengue virus transmission has expanded considerably in tropical and subtropical regions of the globe, and the number of cases of dengue illness reported worldwide has increased more than 30 -fold since the $1960 \mathrm{~s}^{1}$. Only in the last few years did the first dengue vaccine enter a field efficacy trial. The slow pace of vaccine development reflects the unique set of challenges that dengue presents, which are of great relevance to basic and applied immunology (BOX 1). This Review briefly summarizes the key clinical, virological and epidemiological facts about dengue. The primary focus is to review recent studies of antibody and $\mathrm{T}$ cell responses to dengue virus and their association with favourable (protection against infection or illness) or unfavourable (enhancement of disease) clinical outcomes.

\section{Dengue virus}

Dengue virus, the causative agent of dengue, is a small, enveloped virus that contains a single-stranded, positivesense (messenger) RNA genome and is a member of the family Flaviviridae. The term 'dengue virus' actually refers to a group of four genetically and antigenically related viruses that are known as serotypes (DENV-1 to DENV-4). All four dengue virus serotypes cause the same clinical manifestations and show similar patterns of systemic dissemination, with tropism principally for monocytes, macrophages and dendritic cells ${ }^{2,3}$.

Dengue viruses are transmitted to humans by mosquitoes of the genus Aedes. The distribution of Aedes spp. mosquitoes explains the heavy burden of viral transmission in tropical and subtropical areas that encompass more than half of the human population. These regions also include popular destinations for tourists from temperate developed countries, presenting a constant risk for the spread of dengue virus into non-endemic areas ${ }^{4,5}$.

Most dengue virus infections pass with minimal or no symptoms. The classical clinical presentation of dengue virus infection, dengue fever, is an acute illness lasting approximately 4-7 days. Fever, chills and malaise are common but nonspecific. Severe retro-orbital headache and myalgias are more characteristic symptoms of dengue. Other common disease features include leukopenia, thrombocytopenia (sometimes severe) and elevated levels of hepatic transaminases. Symptoms and signs of infection resolve without complications in the vast majority of cases. However, distinctive features of spontaneous bleeding, plasma leakage, or both may appear towards the end of the illness. The dengue plasma-leakage syndrome, named dengue haemorrhagic fever, occurs in only a small percentage of patients, but 
Box 1 |Dengue: challenges to vaccine development

- Multiple viral serotypes

- Incomplete cross-protection

- Viral interference

- Immunological interference

- Animal models are not faithful to human disease

- No validated immunological correlates of immunity (protective or pathological)

is the most important contributor to a severe clinical outcome. Increased vascular permeability in dengue haemorrhagic fever results in decreased circulating plasma volume, haemoconcentration, and pleural and peritoneal effusions, and severe, life-threatening shock can ensue.

The transient nature of plasma leakage, its association with the late febrile phase and the paucity of structural damage to the vasculature in autopsy studies initially suggested that circulating factors were primarily responsible for this phenomenon. Studies have shown that plasma levels of pro-inflammatory and vasoactive cytokines are elevated in patients with dengue haemorrhagic fever before and at the time of plasma leakage, and the degree of elevation correlates with disease severity (reviewed in REF. 6) (BOX 2). As in the case of infection with several other viruses (such as influenza viruses and hantaviruses), the scenario envisioned is that excessive immune activation creates a cascade of cytokine production resulting in increased vascular permeability: a 'cytokine storm'.

Experimental and observational studies indicate that the adaptive immune response to dengue virus has both protective and detrimental aspects. Individuals who have been infected with one dengue virus serotype (primary dengue virus infection) have long-term protective immunity against re-infection with the same serotype. There is also transient resistance to infection with other dengue virus serotypes, but this heterotypic protective immunity lasts only a few months, after which these individuals are once again susceptible to infection with other serotypes (secondary dengue virus infection) ${ }^{7}$. Subsequently, the remaining cross-reactive immune response to other serotypes has the potential to increase the risk for developing dengue haemorrhagic

\section{Box 2 | Plasma markers associated with severe dengue}

\section{Cytokines}

IFNa, IFN $\gamma$, IL-6, IL-8, IL-10, CXCL9, CXCL10, CXCL11, MIF, TNF and VEGF

\section{Soluble receptors}

Soluble CD4, soluble CD8, soluble TNF receptor, soluble IL-2 receptor and IL-1 receptor antagonist

Coagulation factors or endothelial cell markers

Von Willebrand factor, tissue factor, plasminogen activator inhibitor, soluble thrombomodulin and soluble VCAM1; soluble VEGF receptor 2 (inverse association)

CXCL, CXC-chemokine ligand; IFN, interferon; IL, interleukin; MIF, macrophage migration inhibitory factor; TNF, tumour necrosis factor; VCAM1, vascular cell adhesion molecule 1;

VEGF, vascular endothelial growth factor. fever, at least in some individuals. Indeed, several prospective studies found that, in children above the age of 1 year, dengue haemorrhagic fever is 15 -fold to 80 -fold more frequent during secondary dengue virus infection than during primary infection. However, even during primary infection, dengue haemorrhagic fever occurs in a small percentage of patients in this age group ${ }^{8-10}$. Infants born to mothers with established immunity to dengue virus are a special group at high risk for dengue haemorrhagic fever and hospitalization during primary infection in the first year of life ${ }^{11,12}$, as discussed below.

Despite many studies using in vitro and animal models, our knowledge of protective and pathological immune responses to dengue virus relies primarily on clinical and epidemiological studies. Primates are the only vertebrates known to be infected by dengue virus in nature. Both humans and non-human primates are susceptible to dengue virus infection, but the viral strains isolated from humans and non-human primates are genetically distinct, indicating that these transmission cycles diverged in the distant past ${ }^{13}$. Furthermore, dengue haemorrhagic fever is not known to occur in non-human primates in either natural or experimental settings. Laboratory animals, such as mice and other rodents, are susceptible to experimental dengue virus infection, and various disease models have been described in mice. Although these models display some features of human dengue disease, they each have significant limitations (reviewed in REF. 14) (TABLE 1). For example, these models have, for the most part, relied on mouse-adapted viruses that appear to be attenuated with respect to human infection. New models such as 'humanized' mice are promising ${ }^{15,16}$, but have not yet yielded new insights into dengue virus-specific immune responses.

\section{Antibody responses to dengue virus}

Targets of the antibody response to dengue virus. The precursor membrane (pre-M) and envelope (E) structural proteins and non-structural protein 1 (NS1) are the principal targets of the antibody response to dengue virus infection in humans (FIG. 1), as demonstrated by western blotting and the characterization of panels of monoclonal antibodies. Antibody responses have also been detected to other non-structural proteins, including NS3 and NS5. However, these responses are weak, especially in primary dengue virus infections ${ }^{17,18}$. NS3 and NS5 localize exclusively within virus-infected cells, but cell lysis owing to viral cytopathic effect or immune cell-mediated lysis may make these proteins accessible for binding to B cell receptors.

Dengue virus E protein. The most extensive characterization of B cell epitopes has been accomplished for the E glycoprotein, which is the principal surface component of the dengue virion. The three main domains that are bound by antibodies correspond to the three domains in the crystal structure, with multiple epitopes residing within each domain ${ }^{19,20}$ (FIC. 1). Antibodies to all of the epitopes display varying degrees of crossreactivity across the four dengue virus serotypes 
Table 1 | Features of animal models of dengue

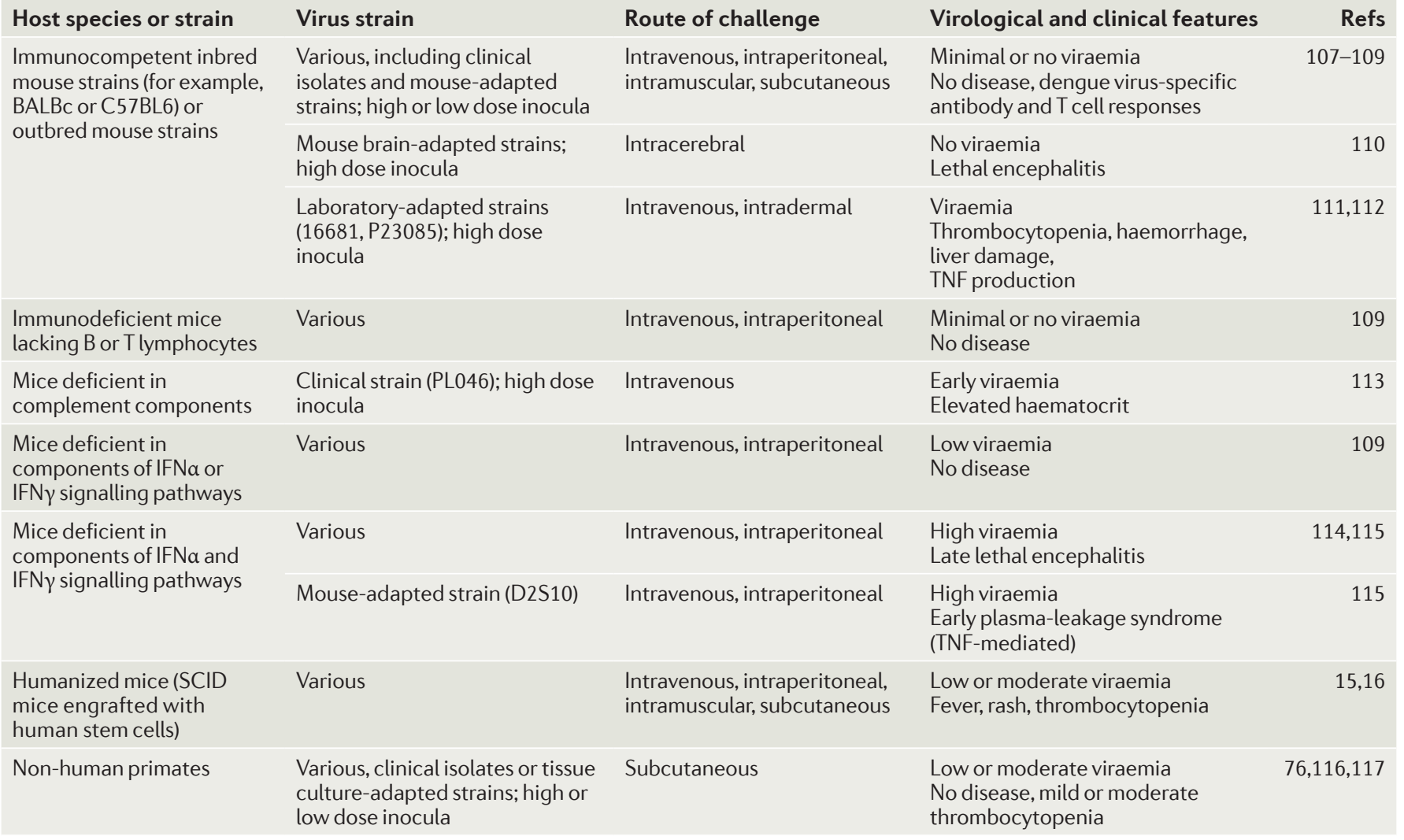

IFN, interferon; SCID, severe combined immunodeficient; TNF, tumour necrosis factor.

Furin

An endoprotease that is important for cleaving and activating precursor proteins such as transforming growth factor- $\beta$ and von Willebrand factor. Viruses, such as HIV, may exploit the activity of host cell furin to promote viral assembly. (and other flaviviruses). Importantly, owing to the dimeric conformation of the E protein on the virion surface and its tight packing in the mature form, not all of the antibody epitopes are equally accessible for antibody binding. For example, under saturating conditions, only 120 of the 180 available E protein domain III epitopes recognized by one monoclonal antibody could be occupied, owing to steric interference at the remaining sites ${ }^{21}$. Another domain III epitope is accessible only during the dynamic rearrangement of the E protein that occurs at physiological temperatures ${ }^{22}$. The fusion loop in domain II of the E protein is almost completely inaccessible in the mature virion, and antibodies to this epitope apparently bind to either immature virions or a post-entry or pre-fusion transition state after low $\mathrm{pH}$-induced rearrangement of the $\mathrm{E}$ protein into a trimeric conformation ${ }^{23}$.

The serological response to the E protein after natural dengue virus infection in humans is highly serotype cross-reactive $^{24}$. Corresponding to the findings using serum, most E protein-specific human monoclonal antibodies were found to bind to more than one dengue virus serotype $\mathrm{e}^{25,26}$. Domain III of the E protein, which contains the putative receptor-binding region that allows the virus to target host cells, is the most variable in amino acid sequence between serotypes. Antibodies specific for this domain show the greatest degree of serotype specificity, but make up a minor component of the overall antibody response during infection ${ }^{24,27}$.
Dengue virus pre-M and NS1 proteins. The pre-M protein forms a heterodimer with the E protein during initial virion assembly and is subsequently cleaved by host cell-expressed furin during the final stage of virion maturation before egress ${ }^{28}$. Following cleavage, the remaining fragment - the M protein — is completely hidden by the $\mathrm{E}$ protein dimers in the mature virion and is inaccessible to antibody binding ${ }^{29}$. However, incomplete cleavage appears to be a common occurrence for dengue virus, at least in vitro, yielding immature and partially mature particles that can be bound by pre-M protein-specific antibodies $^{30}$. Antibodies to the pre-M protein are highly serotype cross-reactive.

The NS1 protein is a glycoprotein that is produced in infected cells but is not incorporated into the virion. NS1 forms a multimeric structure and can be found on the surface of infected cells or can be released as a soluble molecule in cell culture and in vivo ${ }^{31-33}$. Antibodies specific for NS1 are also highly serotype cross-reactive ${ }^{30}$.

Functions of dengue virus-specific antibodies. Antibodies specific for dengue virus proteins mediate a wide range of functions in vitro. Neutralization of infection by dengue virus-specific antibodies can occur through several different mechanisms, including inhibition of binding to cell surface receptors or post-binding inhibition of viral fusion ${ }^{34,35}$. Neutralizing antibodies are directed against the $\mathrm{E}$ protein and include antibodies to nearly all of the epitopes. Neutralization apparently 


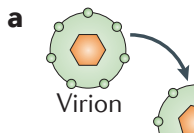

Unknown host receptor for virus

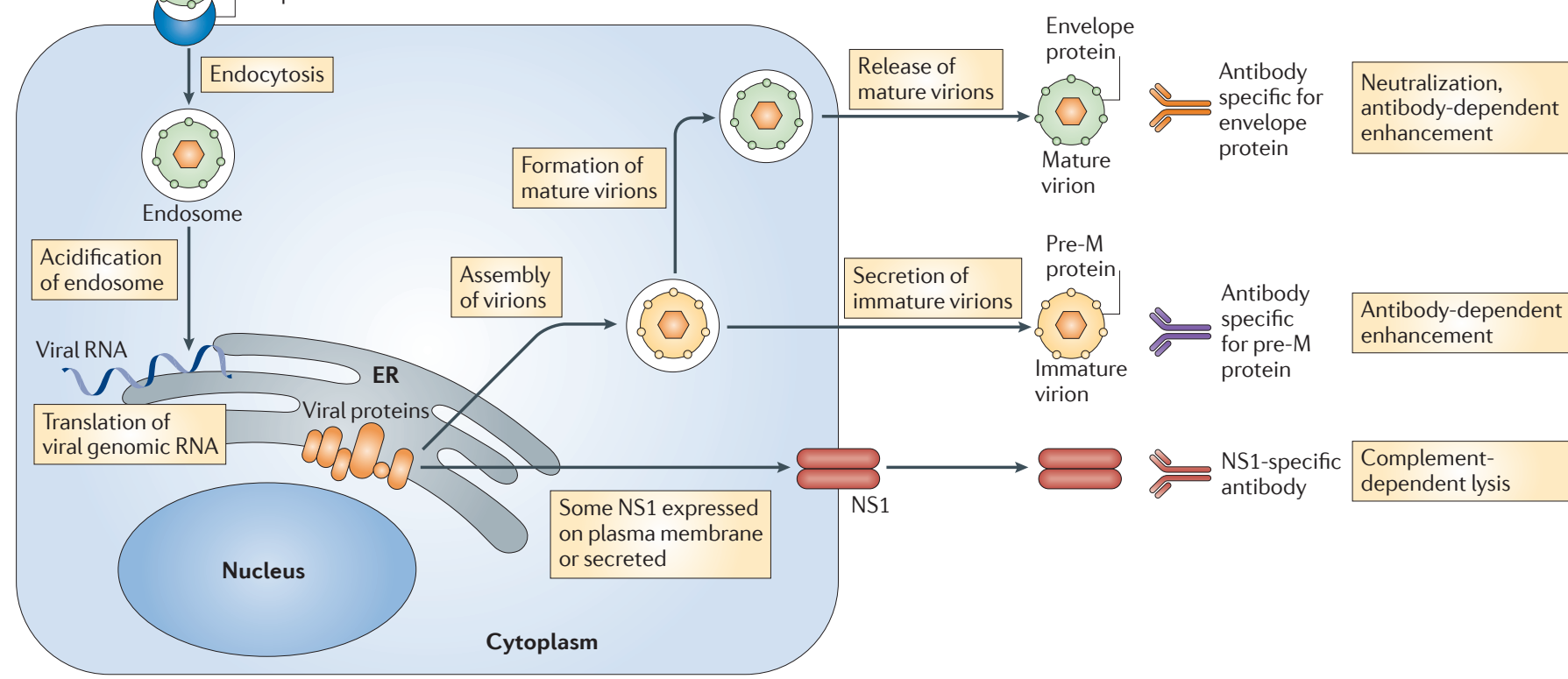

DII
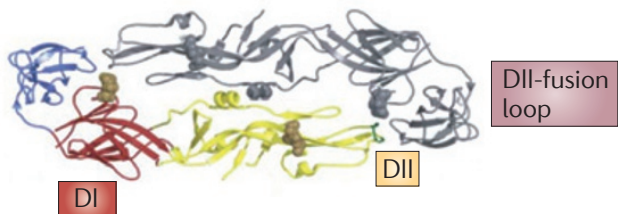
loop

\begin{tabular}{|llll|}
\hline Domain & Function & Serotype specificity & Antibody function \\
\hline I & Hinge region & Cross-reactive $>$ specific & $\begin{array}{l}\text { Weak neutralization } \\
\text { Antibody-dependent } \\
\text { enhancement }\end{array}$ \\
\hline II & $\begin{array}{l}\text { Fusion peptide } \\
\text { dimerization }\end{array}$ & Cross-reactive $>$ specific & $\begin{array}{l}\text { Weak neutralization } \\
\text { Antibody-dependent } \\
\text { enhancement }\end{array}$ \\
\hline III & $\begin{array}{l}\text { Receptor } \\
\text { binding }\end{array}$ & Specific $>$ cross-reactive & $\begin{array}{l}\text { Potent neutralization } \\
\text { Antibody-dependent } \\
\text { enhancement }\end{array}$ \\
\hline
\end{tabular}

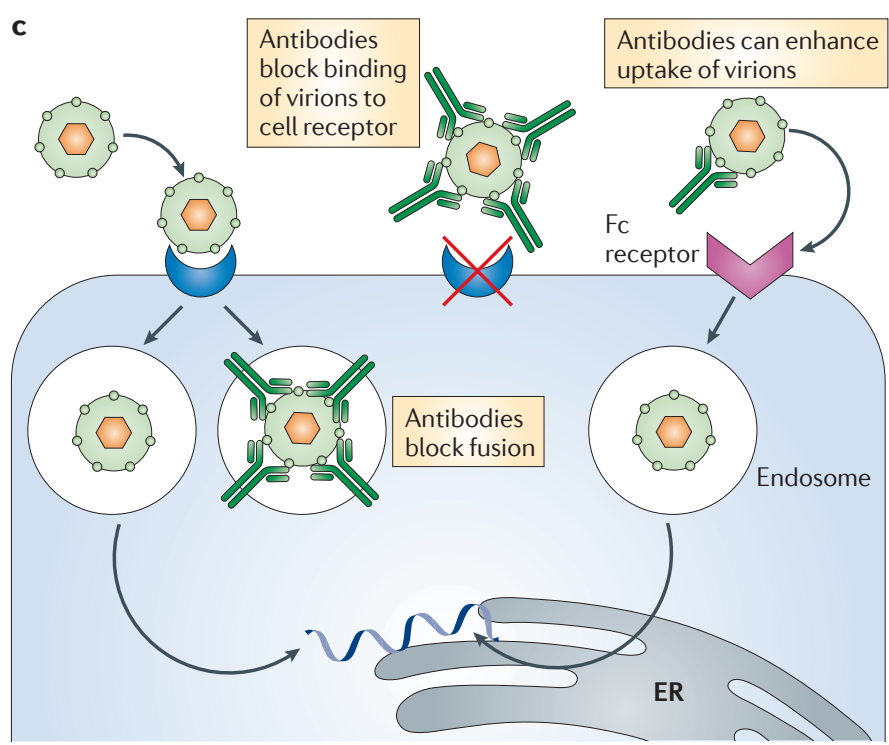

Figure 1 Antibody responses to dengue virus protein targets and antibody functions. a $\mid$ The dengue virus life cycle and sources of antigens are shown. Dengue virions bind to cell surface receptors (these have not been completely characterized), and the virions are internalized through endocytosis. Acidification of the endocytic vescicle leads to rearrangement of the surface envelope (E) glycoprotein, fusion of the viral and vesicle membranes and release of viral RNA into the cytoplasm. Viral genomic RNA is then translated to produce viral proteins in endoplasmic reticulum (ER)-derived membrane structures, and the viral proteins and newly synthesized viral RNA assemble into immature virions within the ER lumen. Cleavage of the viral precursor membrane (pre-M) protein by the host cell enzyme furin leads to the formation of mature virions, which are secreted from the cell. In addition, some of the synthesized non-structural protein 1 (NS1) is expressed on the plasma membrane of the cell or secreted, and some virions are secreted in an immature form. Mature and immature virions induce antibody responses to the E protein, and these antibodies can function in neutralization or in antibody-dependent enhancement of infection. Immature virions also induce antibody responses to the pre-M protein. Antibodies specific for NS1 can interact with membrane-bound NS1 and cause complement-dependent lysis of virus-infected cells. $\mathbf{b}$ |The structure of the dengue virus E glycoprotein ectodomain and characteristics of E protein-specific antibodies are shown. The three domains of the E protein are coloured in red (domain I), yellow (domain II) and blue (domain III). c|The mechanisms of neutralization and enhancement by dengue virus-specific antibodies are shown. At high levels of epitope occupancy, antibodies can block the binding of virions to the cellular receptor or can block fusion at a post-binding stage. At lower epitope occupancy levels, antibodies can enhance the uptake of virions into cells by interacting with immunoglobulin $(\mathrm{Fc})$ receptors. 
requires a minimum occupancy of the available epitopes, which has been estimated to be $\sim 30$ sites per virion ${ }^{36}$. However, measurement of the neutralizing activity of immune sera or monoclonal antibodies is affected by the assay method and by the cell substrate used ${ }^{37}$.

The tropism of dengue virus for monocytes and macrophages, which both express receptors for immunoglobulins, also creates the opportunity for dengue virus-specific antibodies to enhance viral entry, a phenomenon termed 'antibody-dependent enhancement of infection'. Antibody-dependent enhancement of infection occurs because host cell uptake of virus-antibody complexes is more efficient than the entry of free virus through the host cell-expressed receptor(s). Antibodydependent enhancement of infection can be mediated by $\mathrm{E}$ protein-specific antibodies under conditions of low antibody concentration or low antibody avidity, when the number of antibody molecules bound per virion is below the threshold necessary for neutralization of the virus $^{36}$. Antibody-dependent enhancement can also be mediated by pre-M protein-specific antibodies ${ }^{30}$. In this case, uncleaved pre-M protein on immature or partially mature virions is targeted by the host antibody response, and pre-M protein cleavage and virion maturation may occur as the virus-antibody complexes are taken up by the host cell. Infection of monocytes by virus-antibody complexes can also alter cellular responses to infection, including the upregulation of IL-10 production in genetically predisposed individuals ${ }^{38,39}$.

Dengue virus-specific antibodies of the appropriate subclasses can also bind to complement proteins and promote their activation. Fixation of complement to virions by antibodies specific for the pre-M and/or E proteins can inhibit viral infection ${ }^{40}$. Antibodies specific for NS1 on the host cell plasma membrane can also direct complement-mediated lysis of infected cells $^{41}$. NS1-specific antibodies may also contribute to antibody-dependent cellular cytotoxicity ${ }^{42,43}$.

\section{T cell responses to dengue virus}

Targets of the T cell response to dengue virus. Dengue virus proteins are translated from the viral genomic RNA as a single polyprotein, which is subsequently cleaved to yield the three structural proteins and seven non-structural proteins. This coding strategy should generate equimolar amounts of all ten proteins. In keeping with this, $\mathrm{T}$ cell epitopes are found throughout the dengue virus polyprotein ${ }^{44}$ (FIG. 2). These epitopes appear to follow the general principles of $\mathrm{T}$ cell epitope immunogenicity, and they show MHC molecule binding kinetics that are similar to those of other immunodominant viral epitopes. Most of the identified T cell epitopes, for both $\mathrm{CD}^{+}$and $\mathrm{CD} 8^{+} \mathrm{T}$ cells, reside in the NS3 protein, which represents only $\sim 20 \%$ of the dengue virus amino acid coding sequence. Limitations in the availability of reagents may explain the apparent immunodominance of NS3 for T cell recognition, at least in part. However, a recent study using overlapping peptides covering the entire DENV-2 polyprotein also found that the highest ex vivo $\mathrm{T}$ cell responses were directed to the NS3 region ${ }^{45}$.
Given that there is $\sim 70 \%$ amino acid identity between the four dengue virus serotypes, T cell epitopes are highly conserved across serotypes, with the typical epitope having $>6$ residues that are completely conserved and several others that are reasonably well conserved ${ }^{46-49}$. A high percentage of the $\mathrm{T}$ cell clones studied have shown reactivity to more than one dengue virus serotype, although serotype-specific $\mathrm{T}$ cell clones have also been found and some exceptional $\mathrm{T}$ cell clones differentially recognize viral strains within the same serotype $\mathrm{e}^{50}$.

Functions of dengue virus-specific T cells. Dengue virus-specific $\mathrm{T}$ cells recognize virus-infected cells and respond with a diverse set of effector functions, including proliferation, target cell lysis and the production of a range of cytokines (FIG. 2). In vitro, both $\mathrm{CD}^{+}$and $\mathrm{CD} 8^{+}$human $\mathrm{T}$ cells with specificity for dengue virus have demonstrated the capacity to lyse MHC-matched virus-infected cells ${ }^{51,52}$. However, the mechanism of viral peptide presentation by MHC class II molecules in this in vitro system has not been defined. Therefore, the relevance of this in vitro observation to the elimination of virus-infected cells, such as monocytes and macrophages, in vivo is uncertain. A broad array of cytokines is produced by dengue virus-specific $\mathrm{T}$ cells in response to the recognition of peptide-MHC complexes on target cells. For most $\mathrm{T}$ cells studied, the pattern of cytokine production follows a $\mathrm{T}$ helper $1\left(\mathrm{~T}_{\mathrm{H}} 1\right)$ - or $\mathrm{T}_{\mathrm{H}} 0$-like profile. Thus, these $\mathrm{T}$ cells produce interferon- $\gamma$ (IFN $\gamma)$, tumour necrosis factor (TNF), interleukin-2 (IL-2) and CC-chemokine ligand 4 (CCL4; also known as MIP1 $\beta$ ), whereas the production of $\mathrm{T}_{\mathrm{H}} 2$-type cytokines, such as IL-4, is less common $^{46,47,49,53}$.

As is the case for dengue virus-specific antibodies, amino acid differences between serotypes can affect the avidity of the interaction between peptide-MHC complexes on dengue virus-infected cells and the $\mathrm{T}$ cell receptors of individual dengue virus-specific $\mathrm{T}$ cell clones, and this has functional consequences (FIG. 2). The functional avidities of individual dengue virusspecific $\mathrm{T}$ cells have been assessed in vitro mainly by testing $\mathrm{T}$ cell functional responses to graded concentrations of synthetic peptides. T cells recognize and lyse target cells at peptide concentrations lower than those required to stimulate the same $\mathrm{T}$ cells to proliferate ${ }^{53-55}$. Furthermore, the peptide concentrations required to stimulate various cytokine responses differ. The induction of IFN $\gamma$ production requires a relatively high peptide concentration, whereas progressively lower concentrations of peptide are required to induce the production of TNF and CCL4 (REF. 55). Peptide variants found in different dengue virus serotypes act as altered peptide ligands, shifting the peptide dose-response curve towards either increased or decreased responsiveness of the T cell. In our experience, the result is a qualitative change or skewing in the cytokine response profile for many $\mathrm{T}$ cell clones, typically with increased production of inflammatory cytokines (such as TNF and CCL4) and decreased production of IFN $\gamma$ and IL-2 (REFS 46,49,55). 
a

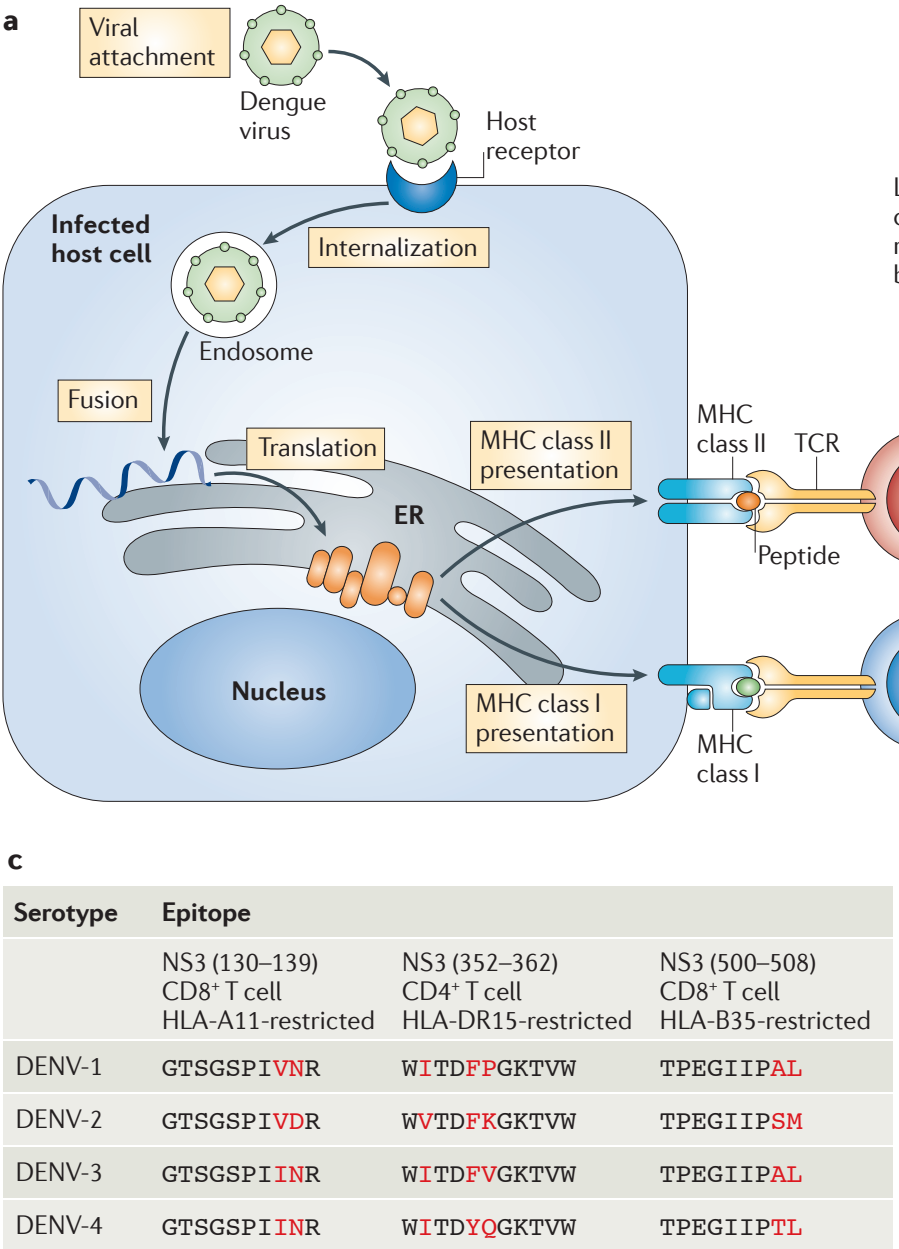

b

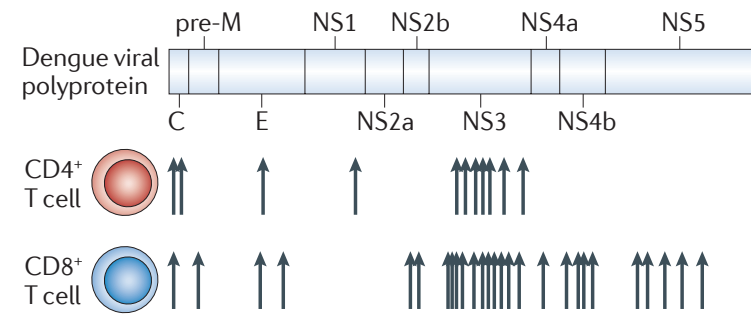

Cytokine production +++

Target cell lysis +

Cytokine production +

Target cell lysis +++

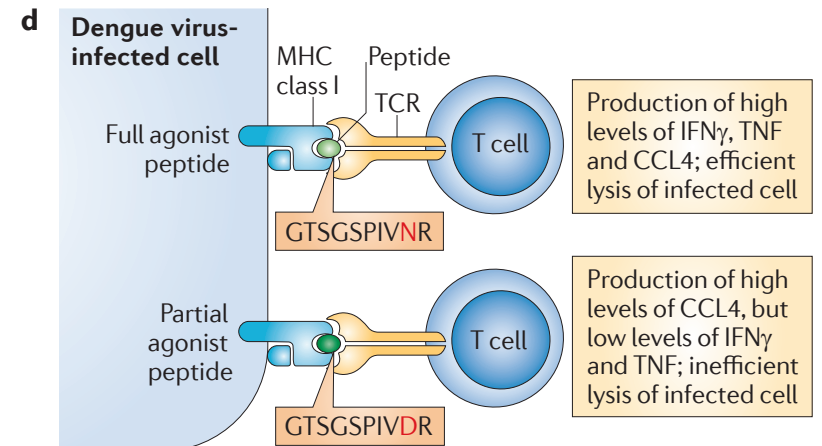

Figure 2 | T cell responses to dengue virus protein targets and T cell effector functions. a $\mid$ The dengue virus life cycle and sources of antigens are shown. Viral attachment, internalization, fusion and translation proceed as described in FIC. 1. Newly synthesized viral proteins enter the MHC class I and II presentation pathways and viral peptide epitopes are presented on the cell surface within the binding groove of MHC molecules. MHC class II molecules present peptides to $\mathrm{CD}^{+} \mathrm{T}$ cells, which principally produce cytokines but are also capable of lysing infected cells. MHC class I molecules present peptides to $C D 8^{+} T$ cells, which principally lyse infected cells but also produce cytokines. $\mathbf{b} \mid$ A schematic of the dengue virus polyprotein is shown at the top and the locations of well-defined epitopes that are recognized by human T cells are marked by arrows. c|Three of the well-defined T cell epitopes are shown to demonstrate the incomplete sequence conservation of typical T cell epitopes. The location of the epitope, its recognition by $\mathrm{CD} 4^{+}$or $\mathrm{CD} 8^{+} \mathrm{T}$ cells and its $\mathrm{HLA}$ restriction are indicated at the top. The predominant sequences for each of the four serotypes are shown. Residues that are completely conserved are shown in black and residues that are not completely conserved are shown in red. $\mathbf{d}$ | Variant epitopes alter the T cell functional response, and the figure shows two examples. The full agonist peptide (top) induces a full range of T cell responses - production of multiple cytokines (for example, IFN $\gamma$, TNF and CCL4) and lysis of the infected cell. A partial agonist peptide varying at one residue (bottom; altered residue in red) induces a skewed functional response, involving production of some cytokines (CCL4 in this example) but little production of other cytokines (such as IFN $\gamma$ ) and inefficient cell lysis. C, capsid protein; CCL4, CC-chemokine ligand 4; E, envelope protein; ER, endoplasmic reticulum; IFN $\gamma$, interferon- $\gamma$; NS, non-structural protein; pre-M, precursor membrane protein; TNF, tumour necrosis factor.

\section{Original antigenic sin}

The potential for an individual to be infected with dengue virus on several different occasions during a lifetime, as described above, is a distinctive clinical and immunological feature of dengue. Importantly, the second and subsequent dengue virus infections always involve different viral serotypes than the previous infection(s), owing to long-lasting homotypic protective immunity against the primary strain. If exceptions to this rule exist in nature, they have yet to be firmly documented. Nevertheless, secondary dengue virus infections differ immunologically from primary infections as a consequence of the fact that the immune response (to the secondary infection) occurs in the context of pre-existing heterotypic immunity that is serotype cross-reactive. Memory B and T cells induced by the prior exposure to a different dengue virus serotype are capable of responding more rapidly than naive cells 
during the acute secondary infection. However, because of sequence diversity between the dengue virus serotypes, the memory B and $\mathrm{T}$ cells that are re-activated during secondary infection may not have optimal avidity for the corresponding epitopes of the new infecting virus. The alteration in immune response, skewed by the 'memory' of the previous infection, is referred to as 'original antigenic sin'.

For antibody responses, affinity maturation occurs within the first few weeks after the onset of primary dengue virus infection ${ }^{56}$. This selects for memory $\mathrm{B}$ cells and plasma cells with the highest specificity and/or affinity for the infecting viral serotype, as evidenced by a wide difference in homotypic versus heterotypic neutralizing antibody titres ${ }^{37,57,58}$. With subsequent dengue virus infection, antibody responses are notably different and are characterized by much higher antibody titres and a broader pattern of neutralization of the four dengue virus serotypes. Titres of antibodies specific for the virus serotype that caused the earlier infection increase substantially, and often remain higher than the titres of antibodies specific for the currently infecting serotype ${ }^{57,59}$, the phenomenon that led to the original coinage of the term original antigenic sin.

For $\mathrm{T}$ cell responses, there are fewer data available comparing primary and subsequent dengue virus infections. Overall, T cell responses after primary dengue virus infections are characterized by higher homotypic than heterotypic responses ${ }^{46,60}$. However, this pattern breaks down at the level of individual $\mathrm{T}$ cell epitopes, and the degree of serotype cross-reactivity is different for the various measurements of $\mathrm{T}$ cell function ${ }^{47,55}$. $\mathrm{T}$ cell responses after secondary infections are highly serotype cross-reactive ${ }^{61}$. Some studies have shown higher responses to the previously encountered dengue virus serotype, consistent with preferential expansion of memory $\mathrm{T}$ cell populations with higher avidity for that serotype than the new infecting serotype ${ }^{48}$. However, our recent studies have revealed a more complex pattern of serotype cross-reactivity, suggesting that other factors that are unique to the individual patient have a strong influence on the $\mathrm{T}$ cell response to secondary infection (H. Friberg, H. Bashyam, T. Toyosaki-Maeda, J. A. Potts, T. Greenough, S. Kalayanarooj, R. V. Gibbons, A. Nisalak, A. Srikiatkhachorn, S. Green, H. A. F. Stephens, A.L.R. and A. Mathew, unpublished observations).

\section{Immune responses associated with protection} Antibody responses associated with protection. Adoptive transfer of immune serum or monoclonal antibodies specific for pre-M, E or NS1 proteins can protect mice from lethal challenge with dengue virus ${ }^{20,41,62,63}$. Similarly, passive transfer of antibodies can protect against infection with dengue virus in non-human primate models ${ }^{64}$. High-avidity antibodies, particularly those specific for epitopes on domain III of the E protein, appear to be most effective at providing protection from infection and/or disease ${ }^{20}$. NS1-specific antibodies mediate complement-dependent lysis of infected cells ${ }^{41}$; however, this may not fully explain their protective effects in vivo ${ }^{65}$.
Assessment of the protective effects of dengue virusspecific antibodies in humans relies on associations from prospective cohort studies, in which exposure to dengue virus is difficult to evaluate. High titres of dengue virusspecific neutralizing antibodies have been associated with a lower likelihood of severe disease during secondary infection ${ }^{66,67}$, but conflicting results have also been reported $^{67}$. Infection and viraemia can clearly occur, even in patients with pre-existing high titres of neutralizing antibodies that can bind to the infecting viral serotype ${ }^{67}$. In these cases, the antibodies detected are presumed to be cross-reactive antibodies from a prior heterotypic dengue virus infection; the available assays are not able to distinguish such heterotypic antibodies from homotypic antibodies, which are thought to provide sterile immunity.

Studies of primary dengue virus infection in infants born to mothers with established immunity to dengue virus provide a means to isolate the effects of passively acquired dengue virus-specific antibodies. The incidence of dengue virus infection, and in particular dengue haemorrhagic fever, is low during the first few months of life, and a minimum neutralizing antibody titre for protection against infection has been suggested ${ }^{12}$. However, the problems with current neutralizing antibody assays (discussed above) confound the interpretation of the results from these studies as well.

T cell responses associated with protection. T cell activity requires the presentation of viral peptides on the surface of infected cells in the context of MHC molecules and, unlike B cells, T cells do not recognize intact virions. Therefore, it would not be possible for dengue virusspecific $\mathrm{T}$ cells to provide sterilizing immunity against viral infection. Nevertheless, the lysis of virus-infected cells and/or the antiviral effects of T cell cytokines (such as IFN $\gamma$ ) could theoretically restrict viral replication and inhibit the development of disease. However, there have been fewer studies of T cell-mediated protection than of antibody-mediated protection.

Adoptive transfer of virus-specific $\mathrm{T}$ cells is one approach to separate the effects of $\mathrm{T}$ cell-mediated immunity from those of humoral immunity, and one study found that dengue virus-specific $\mathrm{CD} 8^{+} \mathrm{T}$ cell clones could partially protect mice from lethal dengue virus challenge ${ }^{68}$. An alternative approach, involving immunization with antigens that induce dengue virusspecific T cells but not neutralizing antibodies, has also shown that $\mathrm{T}$ cells are sufficient to protect mice from lethal infection ${ }^{69-71}$. Most studies of this type have used vaccines designed to induce $\mathrm{CD} 8^{+} \mathrm{T}$ cells, but a study of a particulate capsid protein vaccine found that $\mathrm{CD} 4^{+}$ $\mathrm{T}$ cells can also contribute to protective immunity to dengue virus ${ }^{71}$. Many of these studies have demonstrated that IFN $\gamma$-producing dengue virus-specific T cells are present following immunization, but IFN $\gamma$ was not proven to be essential for the protective effect.

It is more challenging to clearly define a protective role for dengue virus-specific $\mathrm{T}$ cells in humans. Other than congenital antibody deficiencies, there are no clinical scenarios in which memory $\mathrm{T}$ cells are present in the absence of dengue virus-specific antibodies. 
Nevertheless, by collecting blood samples from prospectively enrolled individuals in dengue-endemic areas, it is possible to test for correlations between pre-infection dengue virus-specific $\mathrm{T}$ cell responses and the outcome of infection. So far, these studies have been done only in small study cohorts. One study compared patients who were hospitalized during their subsequent dengue virus infection with patients who were not hospitalized (a decision made independently by the clinician that presumably reflected the severity of disease). The mean $\mathrm{T}$ cell proliferation responses and cytokine secretion levels prior to infection were similar in the two cohorts, but IFN $\gamma$ production in response to a broad range of serotypes was significantly more common among patients who were not hospitalized ${ }^{72}$. In a separate group of subjects, the frequencies of cytokine-producing $\mathrm{CD} 4^{+} \mathrm{T}$ cells were higher in patients who experienced a subclinical secondary dengue virus infection than in patients who had a symptomatic secondary infection ${ }^{73}$. In addition, a clinical study of recipients of a candidate tetravalent vaccine who were subsequently challenged with infectious dengue virus found that protective immunity (that is, protection from the development of fever or viraemia during challenge) was associated with sustained in vitro IFN $\gamma$ responses mediated by dengue virus-specific $\mathrm{T}$ cells in blood samples collected during the acute phase ${ }^{74}$. However, pre-challenge blood samples did not identify an immunological correlate of outcome in that study.

A protective effect of dengue virus-specific $\mathrm{T}$ cells can also be inferred from studies of HLA associations with dengue disease. Specific HLA alleles were found to be significantly more common among patients with dengue fever than among those with the more severe dengue haemorrhagic fever in one or more studies reviewed in REF. 75. These alleles (or allele groups) included HLA-A ${ }^{\star} 0203$, HLA-A ${ }^{\star} 29$, HLA-A ${ }^{\star} 33$, HLA-B $^{\star} 13$, HLA $^{\star}{ }^{\star} 15$, HLA $^{*}{ }^{\star} 44$, HLA-B ${ }^{\star} 52$, HLA-DRB $1^{\star} 04$, HLA-DRB $1^{\star} 07$ and HLA-DRB $1^{\star} 09$. T cell epitopes have been defined for some of these alleles, but no data are available to implicate specific epitopes in any protective effect.

\section{Immune responses associated with disease}

Antibody responses associated with disease. The wellestablished finding that patients with secondary dengue virus infections have an increased risk for developing dengue haemorrhagic fever provides strong evidence for a pathological side to the dengue virus-specific immune response. This association, juxtaposed with the occurrence of dengue haemorrhagic fever during primary infection in infants under 1 year of age, has focused the attention of many groups on the possible detrimental effects of dengue virus-specific antibodies. Antibody-dependent enhancement of infection provides a straightforward explanation for more severe disease in both settings, as increased infection of immunoglobulin receptor-expressing cells (such as monocytes and macrophages) could explain the greater level of cytokine production that occurs. Antibody-dependent enhancement of dengue virus infection has been demonstrated in vivo using adoptive transfer of dengue virus-specific immunoglobulins; in monkeys, increased virus titres were observed ${ }^{76}$ and, in immunodeficient mice, increased virus titres and mortality were noted ${ }^{77}$. Moreover, infection-enhancing activity was displayed in vitro by sera obtained from subjects who had serological evidence of previous dengue virus infection (prior to secondary dengue virus infection) and by sera from the mothers of infants who later developed dengue haemorrhagic fever during primary dengue virus infection ${ }^{12,66,78-80}$.

Several lines of evidence question whether antibodydependent enhancement of infection alone is sufficient to explain dengue haemorrhagic fever. First, dengue haemorrhagic fever can occur, albeit at a lower frequency, in primary dengue virus infection in older children and adults, and this indicates that antibody-dependent enhancement is not required for the development of plasma leakage ${ }^{81}$. Second, although average peak virus titres are higher in patients with dengue haemorrhagic fever than in patients with dengue fever, virus titres are several logs below peak levels by the time plasma leakage occurs, and there are many patients who have high virus titres but do not develop plasma leakage ${ }^{81-83}$. These findings suggest that increased viraemia alone is not the direct cause of plasma leakage and that other mechanisms are involved in the cytokine storm phenomenon. Last, several prospective studies have found no significant difference in the antibody-dependent infection-enhancing activity of pre-infection serum from patients who did and did not subsequently develop dengue haemorrhagic fever. Thus, antibody-dependent infection-enhancing activity is not useful as a correlate of disease risk ${ }^{12,80}$.

Several other mechanisms by which dengue virusspecific antibodies could contribute to dengue haemorrhagic fever have been proposed. First, virus-antibody or antigen-antibody complexes could activate the complement pathway. Complement activation is a feature of severe dengue infection, and is temporally related to plasma leakage ${ }^{84,85}$. Second, some dengue virus-specific antibodies have been reported to cross-react with host proteins. For example, antibodies generated against the E protein can recognize plasminogen ${ }^{86}$, and NS1-specific antibodies can also bind to various coagulation or endothelial cell-expressed proteins ${ }^{87}$. Antibody binding to one or more of these host proteins could activate coagulation pathways and/or alter endothelial cell function. However, the kinetics of these antibody responses do not correspond well to the timing of plasma leakage.

$T$ cell responses associated with disease. T cell-derived cytokines have pleiotropic effects, including the induction or enhancement of inflammation and the alteration of vascular permeability. Infusion of either IL-2 or TNF can induce systemic vascular leakage ${ }^{88,89}$, and this provides a theoretical basis for the participation of T cells in the pathogenesis of dengue haemorrhagic fever. The evidence of increased levels of cytokines in the circulation of patients with dengue haemorrhagic fever has been noted above. In support of a role for T cells in this cytokine production in vivo, studies have also shown high levels of soluble forms of T cell surface molecules (such as 
soluble CD4, soluble CD8 and soluble IL-2R) in patients with dengue haemorrhagic fever, and this is indicative of $\mathrm{T}$ cell activation ${ }^{83,90,91}$. In addition, the frequency of activated $\mathrm{T}$ cells in vivo (as determined by expression of the activation marker CD69 on peripheral blood T cells) was found to be significantly higher in patients with dengue haemorrhagic fever than in those with dengue fever ${ }^{92}$.

Increased $\mathrm{T}$ cell activation and cytokine production have been reported in patients with dengue haemorrhagic fever during both primary and secondary dengue virus infections ${ }^{92,93}$. Thus, it is unclear to what extent the level of $\mathrm{T}$ cell activation during acute infection is determined by factors existing before the acute infection (such as memory $\mathrm{T}$ cells induced by earlier infections or immunizations) versus early events during the acute infection (such as the amount of virus injected by the mosquito or the replication kinetics of the infecting virus strain). Studies of acute dengue virus infection show that, by the time the infection is detected, the immunological events associated with plasma leakage are already in progress ${ }^{83,91,94}$. Prospective collection of blood samples before secondary dengue virus infections is the only way to address this issue. In one cohort study (mentioned above), T cells from a subset of patients showed in vitro TNF secretion in response to stimulation with dengue virus antigens, and
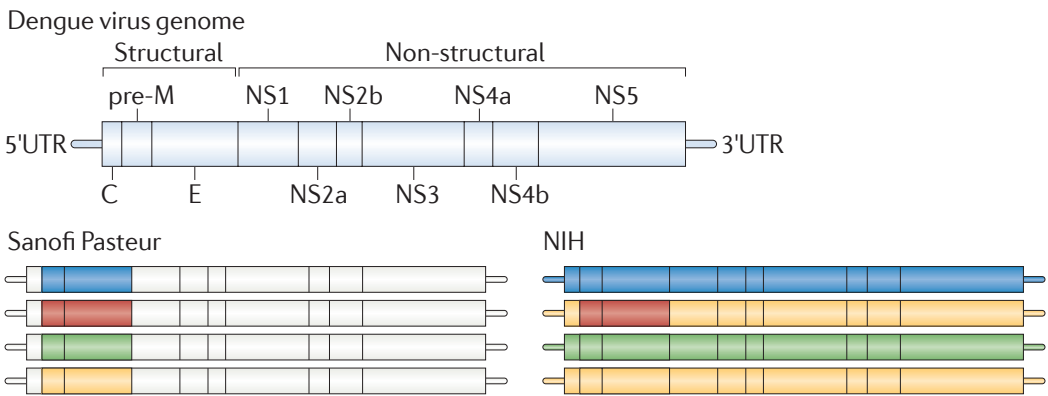

\section{CDC-Inviragen}

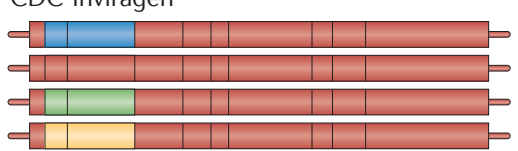

Merck/Hawaii Biotech

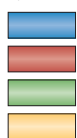

DNA or heterologous

DENV-1 genome

DENV-2 genome

DENV-3 genome

DENV-4 genome

viral vec

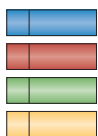

Yellow fever

virus genome

Figure 3 | The antigenic make-up of various dengue vaccine approaches in

development. A schematic of the dengue virus genome is shown at the top. The Sanofi

Pasteur vaccine contains four chimeric live flaviviruses, each derived from the yellow fever virus genome with the precursor membrane (pre-M) and envelope (E) gene segments replaced by the corresponding gene segments of each of the four dengue virus serotypes (DENV-1 to DENV-4). The US National Institutes of Health (NIH) vaccine contains a mixture of four recombinant dengue virus genomes; the DENV-2 component is a chimeric dengue virus derived from a DENV-4 genome with pre-M and $\mathrm{E}$ gene segments replaced by those of DENV-2. This vaccine also contains attenuating mutations in the 3 ' untranslated region (UTR) that are not shown in the figure. The CDC/Inviragen vaccine contains a mixture of four recombinant DENV-2 genomes; the DENV-1, DENV-3 and DENV-4 components are chimeric dengue virus genomes. The Merck/Hawaii Biotech vaccine is a recombinant protein vaccine containing the E glycoprotein ectodomains of all four dengue virus serotypes. Other vaccines in development use plasmids or heterologous viral vectors to express only the pre-M and $\mathrm{E}$ gene segments of all four dengue virus serotypes. all of these patients were hospitalized by the treating physician, which suggests a greater clinical severity of illness ${ }^{72}$. These findings imply that specific patterns of memory $\mathrm{T}$ cell responses represent a higher risk for more severe disease, but that there may not be one single predisposing factor that explains all severe dengue cases.

As with protective T cell responses, HLA association studies provide additional evidence that $\mathrm{T}$ cell responses can determine a more severe clinical outcome. Several studies have found an association between dengue haemorrhagic fever and HLA-A2 in ethnic Thais ${ }^{95,96}$, although a study in Vietnamese patients failed to show this link ${ }^{97}$. A more detailed study using high-resolution molecular typing of HLA-A2 found a positive association of dengue haemorrhagic fever with HLA- $A^{\star} 0207$ but a negative association with HLA-A*0203 (REF. 96). Other allele groups that were more frequent in patients with dengue haemorrhagic fever than in patients with dengue fever or in control individuals in at least one study include HLA-A ${ }^{\star} 01, \mathrm{HLA}-\mathrm{A}^{\star} 24, \mathrm{HLA}-\mathrm{A}^{\star} 31, \mathrm{HLA} \mathrm{B}^{\star} 15$, HLA-B ${ }^{\star} 46$ and HLA-B ${ }^{\star} 51$ (reviewed in REF. 75). However, none of these associations has been defined to the level of a specific epitope or mechanism.

\section{Immune responses to dengue vaccines}

Several different approaches to the development of vaccines against dengue have been followed, and these have significant differences in their immunological targets (FIG. 3). Live attenuated dengue virus strains were developed by several groups through serial passage in tissue culture. Immunization with individual live attenuated strains of dengue virus induced antibody and $\mathrm{T}$ cell responses comparable in titre and specificity to natural infection ${ }^{98-101}$. However, simultaneous administration of strains of all four dengue virus serotypes as tetravalent vaccines introduced additional complications. Inter-serotype interference at the level of viral replication resulted in a dominant viraemia with one or two of the four viruses, and immunodominance further skewed both the antibody and $\mathrm{T}$ cell responses such that responses to one or more serotypes were suboptimal. Predictors of the pattern of immune response to the tetravalent formulations have not been defined. Given these challenges, as well as undesirable safety profiles, the vaccine candidates that were based on empirically attenuated dengue virus strains have been abandoned.

The development of technology to generate synthetic flavivirus genomes and to manipulate them to create viable chimeric viruses has permitted the generation of viruses that have specific attenuating mutations and express desired immunological targets. One such approach has used the genome of the vaccine strain of yellow fever virus to produce a tetravalent dengue virus vaccine, by replacing the gene segments encoding the yellow fever virus pre-M and $\mathrm{E}$ proteins with the corresponding segments from representative strains of each of the four dengue virus serotypes ${ }^{102}$. This vaccine induces dengue virus-specific antibody and $\mathrm{T}$ cell responses directed against epitopes on the pre-M and E proteins, although responses to the four serotypes are not equal because interference and immunodominance 
Anamnestic response The enhanced immune response that occurs against an antigen as a result of previous host exposure to a related antigen. still occur ${ }^{103}$. This vaccine also induces $\mathrm{T}$ cell responses to the yellow fever virus non-structural proteins ${ }^{104}$ and presumably antibody responses to yellow fever virus non-structural proteins as well. Most dengue virus and yellow fever virus epitopes are highly divergent and, not unexpectedly, antibody and $\mathrm{T}$ cell responses to the dengue virus non-structural proteins following administration of this vaccine are low to undetectable. However, some degree of cross-reactivity with dengue virus and other flaviviruses may explain the anamnestic response observed to subsequent flavivirus immunizations.

The use of attenuated dengue virus strains as the genetic backbone for the construction of denguedengue chimaeras has yielded a hybrid vaccine approach, in which single-serotype strains and mixed-serotype chimeric strains are combined to produce a tetravalent formulation $^{105,106}$ (FIG. 3). Clinical testing is still in an early stage, and the antibody and $\mathrm{T}$ cell responses to the tetravalent vaccine have not been reported. However, this vaccine might be expected to express different epitopes in varying proportions, depending on the 'dose' of each

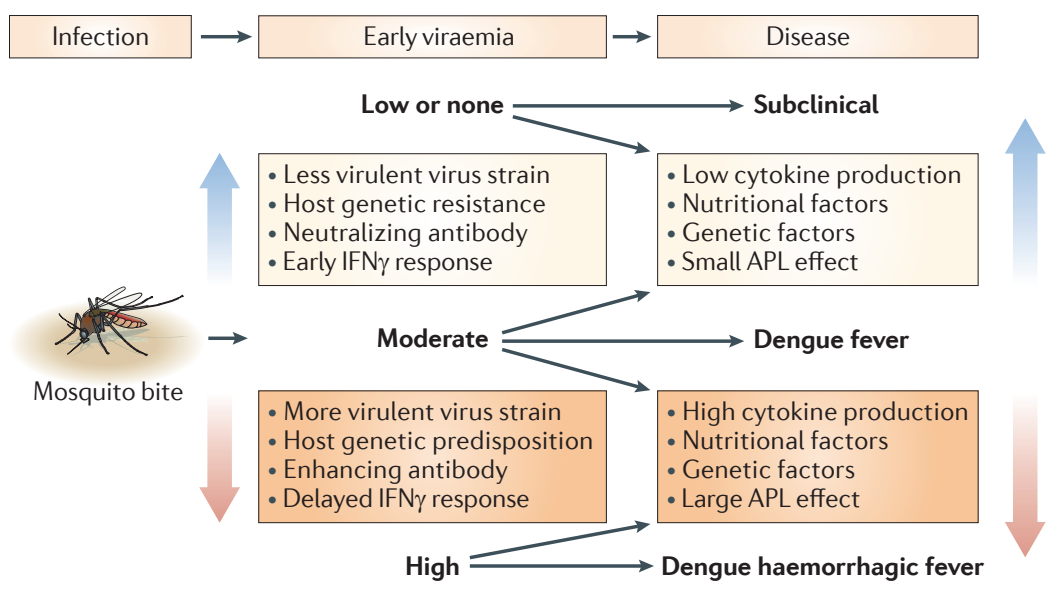

Figure 4 | A balance between favourable and unfavourable factors determines the clinical outcome of dengue virus infection. Virus inoculation by Aedes aegypti mosquitoes results in viral dissemination, which in turn results in disease manifestations; high virus titres are necessary but not sufficient to cause severe disease. Viral and host factors affect early viral replication and influence the level of viraemia. Other host factors affect the levels of production of various cytokines (such as interferon- $\gamma(\mathrm{IFN} \gamma)$ and tumour necrosis factor (TNF)) and determine the severity of illness for any given level of viraemia. APL, altered peptide ligand. segment of the genome. The impact of this variation, in combination with the effects of interference and immunodominance seen with other live dengue vaccines, will need to be determined as the clinical studies progress.

Other candidate dengue vaccines in development express only subsets of the dengue virus proteome. These include protein subunit vaccines (such as those that use recombinant $\mathrm{E}$ protein); inactivated whole virions that contain only structural proteins; nucleic acid vaccines that encode pre-M and E proteins with or without NS1; and heterologous viral vectors (such as measles virus) that express these same dengue virus genome segments. Most such vaccines have been designed specifically to induce dengue virus-specific neutralizing antibodies. Preclinical testing has yielded promising results, but clinical trials of these vaccines are in an early stage or have yet to begin.

\section{Conclusions and perspectives}

The available evidence suggests that the outcome of dengue virus infection depends on the balance between favourable and unfavourable immune responses, the former providing control of viral replication and the latter enhancing the inflammatory and vascular permeability response to a given level of antigen expression (FIG. 4). Host genetics, the sequence of infection with different dengue virus serotypes and strains, and probably other factors specific to the individual patient modulate the pattern of the immune response, particularly with regard to cross-reactivity with other dengue virus serotypes not yet encountered.

The lack of reliable immunological markers for either protective or pathological immune responses to dengue virus represents a major gap in our current knowledge base and should be a high priority as clinical trials of dengue vaccines progress into the phase of testing vaccine efficacy. Whether the same markers will apply to all of the different vaccine approaches is also an open question and will necessitate a cautious approach to extrapolating from studies of natural infection or other vaccines. New animal models may help to address these questions experimentally, but they are not yet at the stage at which they can provide reliable information to guide vaccine development or testing. For the foreseeable future, carefully designed clinical studies will remain essential to advance efforts to reduce the global impact of dengue.
1. World Health Organization. Dengue: guidelines for diagnosis, treatment, prevention and control. New edition 2009. WHO [online], http://whqlibdoc.who.int/ publications/2009/9789241547871_eng.pdf (2009).

2. Jessie, K., Fong, M. Y., Devi, S., Lam, S. K. \& Wong, K. T. Localization of dengue virus in naturally infected human tissues, by immunohistochemistry and in situ hybridization. J. Infect. Dis. 189, 1411-1418 (2004).

The most recent published study to identify dengue virus-infected cells in human tissues, and the only one to detect both viral antigens and RNA.

3. Durbin, A. P. et al. Phenotyping of peripheral blood mononuclear cells during acute dengue illness demonstrates infection and increased activation of monocytes in severe cases compared to classic dengue fever. Virology 376, 429-435 (2008)
4. La Ruche, G. et al. First two autochthonous dengue virus infections in metropolitan France September 2010. Euro Surveill. 15, 19676 (2010).

5. Centers for Disease Control and Prevention. Locally acquired dengue - Key West, Florida, 2009-2010. MMWR Morb. Mortal. Wkly Rep. 59, 577-581 (2010).

6. Srikiatkhachorn, A. \& Green, S. Markers of dengue disease severity. Curr. Top. Microbiol. Immunol. 338 67-82 (2010).

7. Sabin, A. B. Research on dengue during World War II. Am. J. Trop. Med. Hyg. 1, 30-50 (1952).

8. Burke, D. S., Nisalak, A., Johnson, D. E. \& Scott, R. M. A prospective study of dengue infections in Bangkok. Am. J. Trop. Med. Hyg. 38, 172-180 (1988).

9. Thein, S. et al. Risk factors in dengue shock syndrome. Am. J. Trop. Med. Hyg. 56, 566-572 (1997).
10. Graham, R. R. et al. A prospective seroepidemiologic study on dengue in children four to nine years of age in Yogyakarta, Indonesia I. studies in 1995-1996. Am. J. Trop. Med. Hyg. 61, 412-419 (1999).

11. Halstead, S. B., Scanlon, J. E., Umpaivit, P. \& Udomsakdi, S. Dengue and chikungunya virus infection in man in Thailand, 1962-1964 IV. Epidemiologic studies in the Bangkok metropolitan area. Am. J. Trop. Med. Hyg. 18, 997-1021 (1969).

12 Libraty, D. H et al A prospective nested case-control study of dengue in infants: rethinking and refining the antibody-dependent enhancement dengue hemorrhagic fever model. PLoS Med. 6, e 1000171 (2009).

13. Wang, E. et al. Evolutionary relationships of endemic epidemic and sylvatic dengue viruses. J. Virol. 74 3227-3234 (2000). 
14. Yauch, L. E. \& Shresta, S. Mouse models of dengue virus infection and disease. Antiviral Res. 80, 87-93 (2008).

15. Bente, D. A., Melkus, M. W., Garcia, J. V. \& RicoHesse, R. Dengue fever in humanized NOD/SCID mice. J. Virol. 79, 13797-13799 (2005)

16. Jaiswal, S. et al. Dengue virus infection and virusspecific HLA-A2 restricted immune responses in humanized NOD-scid IL2r $\gamma^{\text {null }}$ mice. PLoS ONE 4 e7251 (2009)

17. Valdes, K. et al. Human dengue antibodies against structural and nonstructural proteins. Clin. Diagn. Lab. Immunol. 7, 856-857 (2000)

18. Churdboonchart, V., Bhamarapravati, N. Peampramprecha, S. \& Sirinavin, S. Antibodies against dengue viral proteins in primary and secondary dengue hemorrhagic fever. Am. J. Trop. Med. Hyg. 44, 481-493 (1991)

19. Roehrig, J. T., Bolin, R. A. \& Kelly, R. G. Monoclonal antibody mapping of the envelope glycoprotein of the dengue 2 virus, Jamaica. Virology 246, 317-328 (1998).

20. Sukupolvi-Petty, S. et al. Structure and function analysis of therapeutic monoclonal antibodies against dengue virus type 2. J. Virol. 84, 9227-9239 (2010).

21. Kaufmann, B. et al. West Nile virus in complex with the Fab fragment of a neutralizing monoclonal antibody. Proc. Natl Acad. Sci. USA 103, 12400-12404 (2006)

22. Lok, S. M. et al. Binding of a neutralizing antibody to dengue virus alters the arrangement of surface glycoproteins. Nature Struct. Mol. Biol. 15, 312-317 (2008).

A crystallographic study of antibody-bound virus, suggesting novel dynamics of the virion particle that could make cryptic epitopes accessible.

23. Cherrier, M. V. et al. Structural basis for the preferential recognition of immature flaviviruses by a fusion-loop antibody. EMBO J. 28, 3269-3276 (2009).

24. Lai, C. Y. et al. Antibodies to envelope glycoprotein of dengue virus during the natural course of infection are predominantly cross-reactive and recognize epitopes containing highly conserved residues at the fusion loop of domain II. J. Virol. 82, 6631-6643 (2008).

25. Schieffelin, J. S. et al. Neutralizing and nonneutralizing monoclonal antibodies against dengue virus $\mathrm{E}$ protein derived from a naturally infected patient. Virol. J. 7, 28 (2010).

26. Beltramello, M. et al. The human immune response to dengue virus is dominated by highly cross-reactive antibodies endowed with neutralizing and enhancing activity. Cell Host Microbe 8, 271-283 (2010).

27. Wahala, W. M., Kraus, A. A., Haymore, L. B. Accavitti-Loper, M. A. \& de Silva, A. M. Dengue virus neutralization by human immune sera: role of envelope protein domain III-reactive antibody. Virology 392, 103-113 (2009).

28 Stadler, K., Allison, S. L., Schalich, J. \& Heinz, F. X Proteolytic activation of tick-borne encephalitis virus by furin. J. Virol. 71, 8475-8481 (1997).

29. Kuhn, R. J. et al. Structure of dengue virus: implications for flavivirus organization, maturation, and fusion. Cell 108, 717-725 (2002).

30. Dejnirattisai, W. et al. Cross-reacting antibodies enhance dengue virus infection in humans. Science 328, 745-748 (2010)

This study characterized a large number of human monoclonal antibodies, demonstrating a high frequency of pre-M-specific antibodies that could enhance infection by immature dengue virions.

31. Jacobs, M. G., Robinson, P. J., Bletchly, C., Mackenzie, J. M. \& Young, P. R. Dengue virus nonstructural protein 1 is expressed in glycosyl-phosphatidylinositol-linked form that is capable of signal transduction. FASEB J. 14 1603-1610 (2000)

32. Young, P. R., Hilditch, P. A., Bletchly, C. \& Halloran, W. An antigen capture enzyme-linked immunosorbent assay reveals high levels of the dengue virus protein NS1 in the sera of infected patients. J. Clin. Microbiol 38, 1053-1057 (2000)

33 Libraty, D. H. et al. High circulating levels of the dengue virus nonstructural protein NS1 early in dengue illness correlate with the development of dengue hemorrhagic fever. J. Infect. Dis. 186, 1165-1168 (2002)

34. Nybakken, G. E. et al. Structural basis of West Nile virus neutralization by a therapeutic antibody. Nature 437, 764-769 (2005).
35. Vogt, M. R. et al. Human monoclonal antibodies against West Nile virus induced by natural infection neutralize at a postattachment step. J. Virol. 83 6494-6507 (2009)

36. Pierson, T. C. et al. The stoichiometry of antibodymediated neutralization and enhancement of West Nile virus infection. Cell Host Microbe 1, 135-145 (2007).

37. Thomas, S. J. et al. Dengue plaque reduction neutralization test (PRNT) in primary and secondary dengue virus infections: how alterations in assay conditions impact performance. Am. J. Trop. Med. Hyg. 81, 825-833 (2009)

38. Ubol, S., Phuklia, W., Kalayanarooj, S. \& Modhiran, N. Mechanisms of immune evasion induced by a complex of dengue virus and preexisting enhancing antibodies. J. Infect. Dis. 201, 923-935 (2010).

39. Boonnak, K., Dambach, K. M., Donofrio, G. C. Tassaneetrithep, B. \& Marovich, M. A. Cell type specificity and host genetic polymorphisms influence antibody-dependent enhancement of dengue virus infection. J. Virol. 85, 1671-1683 (2011). This study shows that antibody-bound dengue virus can alter the host cell cytokine response, and that this effect is genetically determined.

40. Mehlhop, E. et al. Complement protein C1q inhibits antibody-dependent enhancement of flavivirus infection in an IgG subclass-specific manner. Cell Host Microbe 2, 417-426 (2007)

41. Schlesinger, J. J., Brandriss, M. W. \& Walsh, E. E. Protection of mice against dengue 2 virus encephalitis by immunization with the dengue 2 virus nonstructural glycoprotein NS1. J. Gen. Virol. 68 853-857 (1987).

42. Garcia, G. et al. Antibodies from patients with dengue viral infection mediate cellular cytotoxicity. J. Clin. Virol. 37, 53-57 (2006)

43. Laoprasopwattana, K. et al. Antibody dependent cellular cytotoxicity in pre-secondary dengue virus serotype 3 (DV3) but not in DV2 infection plasma samples inversely correlated with viremia levels. J. Infect. Dis. 195, 1108-1116 (2007).

44. Vaughan, K., Greenbaum, J., Blythe, M., Peters, B. \& Sette, A. Meta-analysis of all immune epitope data in the Flavivirus genus: inventory of current immune epitope data status in the context of virus immunity and immunopathology. Viral Immunol. 23, 259-284 (2010).

45. Duangchinda, T. et al. Immunodominant T-cell responses to dengue virus NS3 are associated with DHF. Proc. Natl Acad. Sci. USA 107, 16922-16927 (2010)

46. Mangada, M. M. \& Rothman, A. L. Altered cytokine responses of dengue-specific $C D 4^{+} \mathrm{T}$ cells to heterologous serotypes. J. Immunol. 175, 2676-2683 (2005).

This study demonstrated that epitopes from heterologous dengue virus serotypes induce different $\mathrm{T}$ cell functional response from the original serotype with which an individual had been infected.

47. Bashyam, H. S., Green, S. \& Rothman, A. L. Dengue virus-reactive $\mathrm{CD}^{+} \mathrm{T}$ cells display quantitative and qualitative differences in their response to variant epitopes of heterologous viral serotypes. J. Immunol. 176, 2817-2824 (2006).

48. Mongkolsapaya, J. et al. Original antigenic sin and apoptosis in the pathogenesis of dengue hemorrhagic fever. Nature Med. 9, 921-927 (2003).

This study demonstrated high frequencies of dengue epitope-specific T cells in patients with secondary infection, and provided evidence that these cells were originally induced by the previous (primary) dengue virus infection.

49. Imrie, A et al. Differential functional avidity of dengue virus-specific T-cell clones for variant peptides representing heterologous and previously encountered serotypes. J. Virol. 81, 10081-10091 (2007)

50. Rothman, A. L. \& Ennis, F. A. in Dengue Tropical Medicine: Science and Practice (ed. Halstead, S. B.) 257-283 (Imperial College Press, London, 2008)

51. Kurane, I., Meager, A. \& Ennis, F. A. Dengue virusspecific human T cell clones. Serotype crossreactive proliferation, interferon $\gamma$ production, and cytotoxic activity. J. Exp. Med. 170, 763-775 (1989).

52. Bukowski, J. F. et al. Dengue virus-specific cross reactive $\mathrm{CD}^{+}{ }^{+}$human cytotoxic $\mathrm{T}$ lymphocytes. J. Virol. 63, 5086-5091 (1989).

53. Dong, T. et al. High pro-inflammatory cytokine secretion and loss of high avidity cross-reactive cytotoxic T-cells during the course of secondary dengue virus infection. PLOS ONE 2, e 1192 (2007).

54. Zivny, J. et al. Partial agonist effect influences the CTL response to a heterologous dengue virus serotype. J. Immunol. 163, 2754-2760 (1999).

55. Friberg, H. et al. Memory CD8 ${ }^{+} \mathrm{T}$ cells from naturally acquired primary dengue virus infection are highly cross-reactive. Immunol. Cell Biol. 89, 122-129 (2011).

56. Matheus, S. et al. Discrimination between primary and secondary dengue virus infection by an immunoglobulin $\mathrm{G}$ avidity test using a single acutephase serum sample. J. Clin. Microbiol. 43, 2793-2797 (2005).

57. Halstead, S. B., Rojanasuphot, S. \& Sangkawibha, N. Original antigenic sin in dengue. Am. J. Trop. Med. Hyg. 32, 154-156 (1983).

58. van Panhuis, W. G. et al. Inferring the serotype associated with dengue virus infections on the basis of pre- and postinfection neutralizing antibody titers. J. Infect. Dis. 202, 1002-1010 (2010).

59. Midgley, C. M. et al. An in-depth analysis of origina antigenic sin in dengue virus infection. J. Virol. $\mathbf{8 5}$, 410-421 (2011).

60. Kurane, I. et al. Human T cell responses to dengue virus antigens. Proliferative responses and interferon $\gamma$ production. J. Clin. Invest. 83, 506-513 (1989).

61. Mathew, A. et al. Predominance of HLA-restricted CTL responses to serotype crossreactive epitopes on nonstructural proteins after natural dengue virus infections. J. Virol. 72, 3999-4004 (1998).

62. Kaufman, B. M. et al. Monoclonal antibodies for dengue virus prM glycoprotein protect mice against lethal dengue infection. Am. J. Trop. Med. Hyg. 41, 576-580 (1989).

63. Brien, J. D. et al. Genotype-specific neutralization and protection by antibodies against dengue virus type 3 J. Virol. 84, 10630-10643 (2010).

64. Lai, C. J. et al. Epitope determinants of a chimpanzee dengue virus type 4 (DENV-4) neutralizing antibody and protection against DENV-4 challenge in mice and rhesus monkeys by passively transferred humanized antibody. J. Virol. 81, 12766-12774 (2007)

65. Henchal, E. A., Henchal, L. S. \& Schlesinger, J. J. Synergistic interactions of anti-NS 1 monoclonal antibodies protect passively immunized mice from lethal challenge with dengue 2 virus. J. Gen. Virol. 69 2101-2107 (1988)

66. Kliks, S. C., Nisalak, A., Brandt, W. E., Wahl, L. \& Burke, D. S. Antibody-dependent enhancement of dengue virus growth in human monocytes as a risk factor for dengue hemorrhagic fever. Am. J. Trop. Med. Hyg. 40, 444-451 (1989)

67. Endy, T. P. et al. Relationship of pre-existing dengue virus (DV) neutralizing antibody levels to viremia and disease severity in a prospective cohort study of DV infection in Thailand. J. Infect. Dis. 189, 990-1000 (2004).

This study provides evidence for a protective effect of cross-reactive neutralizing antibodies against viraemia and disease, but the effect may depend on the serotype of dengue virus causing the secondary infection.

68. An, J. et al. Dengue-specific CD8 ${ }^{+} \mathrm{T}$ cells have both protective and pathogenic roles in dengue virus infection. Immunol. Lett. 95, 167-174 (2004)

69. Yauch, L. E. et al. A protective role for dengue virusspecific CD8+ T cells. J. Immunol. 182, 4865-4873 (2009).

70. Gil, L. et al. Recombinant nucleocapsid-like particles from dengue- 2 virus induce protective $\mathrm{CD}_{4}^{+}$and $\mathrm{CD}^{+}$cells against viral encephalitis in mice. Int Immunol. 21, 1175-1183 (2009).

71. Lazo, L. et al. Nucleocapsid-like particles of dengue-2 virus enhance the immune response against a recombinant protein of dengue-4 virus. Arch. Virol. 155, 1587-1595 (2010).

72. Mangada, M. M. et al. Dengue-specific T cell responses in peripheral blood mononuclear cells obtained prior to secondary dengue virus infections in Thai schoolchildren. J. Infect. Dis. 185, 1697-1703 (2002).

73. Hatch, S. et al. Intracellular cytokine production by dengue virus-specific T cells correlates with subclinical secondary infection. J. Infect. Dis. 203, 1282-1291 (2011)

This study provides evidence of a protective effect of pre-existing dengue virus-specific memory T cells against clinical disease during subsequent infection. 
74. Gunther, V. J. et al. A human challenge model for dengue infection reveals a possible protective role for sustained interferon $\gamma$ levels during the acute phase of illness. Vaccine 29, 3895-3904 (2011).

This study of experimental dengue virus challenge in humans suggests a protective role for IFN $\gamma$ responses.

75. Stephens, H. A. HLA and other gene associations with dengue disease severity. Curr. Top. Microbiol. Immunol. 338, 99-114 (2010).

76. Goncalvez, A. P., Engle, R. E., St. Claire, M., Purcell, R. H. \& Lai, C. J. Monoclonal antibody-mediated enhancement of dengue virus infection in vitro and in vivo and strategies for prevention. Proc. Natl Acad. Sci. USA 104, 9422-9427 (2007).

In this study, administration of sub-neutralizing antibodies prior to dengue virus challenge resulted in higher virus titres in rhesus monkeys, demonstrating antibody-dependent enhancement of infection in vivo.

77. Zellweger, R. M., Prestwood, T. R. \& Shresta, S Enhanced infection of liver sinusoidal endothelial cells in a mouse model of antibody-induced severe dengue disease. Cell Host Microbe 7, 128-139 (2010).

This study showed lethal systemic infection in IFN-deficient mice as a potential model of antibody-dependent enhancement of infection.

78. Kliks, S. Antibody-enhanced infection of monocytes as the pathogenetic mechanism for severe dengue illness. AIDS Res. Hum. Retroviruses 6, 993-998 (1990).

79. Kliks, S. C., Nimmanitya, S., Nisalak, A. \& Burke, D. S Evidence that maternal dengue antibodies are important in the development of dengue hemorrhagic fever in infants. Am. J. Trop. Med. Hyg. 38, 411-419 (1988).

80. Laoprasopwattana, K. et al. Dengue virus (DV) enhancing antibody activity in preillness plasma does not predict subsequent disease severity or viremia in secondary DV infection. J. Infect. Dis. 192, 510-519 (2005).

In this study, the activity of pre-infection serum in enhancement of dengue virus infection in vitro was not associated with viraemia or disease during secondary infection.

81. Vaughn, D. W. et al. Dengue viremia titer, antibody response pattern and virus serotype correlate with disease severity. J. Infect. Dis. 181, 2-9 (2000).

82. Vaughn, D. W. et al. Dengue in the early febrile phase viremia and antibody responses. J. Infect. Dis. 176 , 322-330 (1997)

83. Libraty, D. H. et al. Differing influences of viral burden and immune activation on disease severity in secondary dengue 3 virus infections. J. Infect. Dis. 185, 1213-1221 (2002).

This study showed that increased dengue disease severity was associated with both higher virus titres and higher cytokine levels.

84. Malasit, P. Complement and dengue haemorrhagic fever/shock syndrome. Southeast Asian J. Trop. Med. Pub. Health 18, 316-320 (1987).

85. Avirutnan, P. et al. Vascular leakage in severe dengue virus infections: a potential role for the nonstructural viral protein NS1 and complement. J. Infect. Dis. 193 1078-1088 (2006).

86. Markoff, L. J., Innis, B. L., Houghten, R. \& Henchal, L. S. Development of cross-reactive antibodies to plasminogen during the immune response to dengue virus infection. J. Infect. Dis. 164, 294-301 (1991).
87. Falconar, A. K. I. The dengue virus nonstructural-1 protein (NS1) generates antibodies to common epitopes on human blood clotting, integrin/adhesion proteins and binds to human endothelial cells: potential implications in haemorrhagic fever pathogenesis. Arch. Virol. 142, 897-916 (1997). This study found that monoclonal antibodies to the dengue virus NS1 protein show cross-reactivity against human endothelial cell and clotting proteins.

88. Dutcher, J. et al. Kidney cancer: the Cytokine Working Group experience (1986-2001): part II. Management of IL-2 toxicity and studies with other cytokines. Med. Oncol. 18, 209-219 (2001)

89. Yang, G. et al. The dual role of TNF in pulmonary edema. J. Cardiovasc. Dis. Res. 1, 29-36 (2010).

90. Kurane, I. et al. Activation of T lymphocytes in dengue virus infections. High levels of soluble interleukin 2 receptor, soluble CD4, soluble CD8, interleukin 2, and interferon- $\gamma$ in sera of children with dengue. J. Clin. Invest. 88, 1473-1480 (1991).

91. Green, S. et al. Early immune activation in acute dengue is related to development of plasma leakage and disease severity. J. Infect. Dis. 179, 755-762 (1999).

92. Green, S. et al. Early CD69 expression on peripheral blood lymphocytes from children with dengue hemorrhagic fever. J. Infect. Dis. 180, 1429-1435 (1999).

93. Chau, T. N. et al. Dengue in Vietnamese infants results of infection-enhancement assays correlate with age-related disease epidemiology, and cellular immune responses correlate with disease severity. J. Infect. Dis. 198, 516-524 (2008).

94. Green, S. et al. Elevated plasma interleukin-10 levels in acute dengue correlate with disease severity. J. Med. Virol. 59, 329-334 (1999).

95. Chiewsilp, P., Scott, R. M. \& Bhamarapravati, N Histocompatibility antigens and dengue hemorrhagic fever. Am. J. Trop. Med. Hyg. 30, 1100-1105 (1981).

96. Stephens, H. A. et al. HLA-A and -B allele associations with secondary dengue virus infections correlate with disease severity and the infecting viral serotype in ethnic Thais. Tissue Antigens 60, 309-318 (2002).

97. Loke, H. et al. Strong HLA class I-restricted T cell responses in dengue hemorrhagic fever: a doubleedged sword? J. Infect. Dis. 184, 1369-1373 (2001).

98. Sun, W. et al. Phase 2 clinical trial of three formulations of tetravalent live-attenuated dengue vaccine in flavivirus-naive adults. Hum. Vaccin. 5 33-40 (2009).

99. Gwinn, W., Sun, W., Innis, B. L., Caudill, J. \& King, A. D. Serotype-specific $T_{H} 1$ responses in recipients of two doses of candidate live-attenuated dengue virus vaccines. Am. J. Trop. Med. Hyg. 69, 39-47 (2003).

100. Kanesa-thasan, N. et al. Safety and immunogenicity of attenuated dengue virus vaccines (Aventis Pasteur) in human volunteers. Vaccine 19, 3179-3188 (2001)

101. Rothman, A. L. et al. Induction of T lymphocyte responses to dengue virus by a candidate tetravalent live attenuated dengue virus vaccine. Vaccine 19 , 4694-4699 (2001)

102. Guirakhoo, F. et al. Viremia and immunogenicity in nonhuman primates of a tetravalent yellow feverdengue chimeric vaccine: genetic reconstructions, dose adjustment, and antibody responses against wild-type dengue virus isolates. Virology 298 146-159 (2002).
103. Morrison, D. et al. A novel tetravalent dengue vaccine is well tolerated and immunogenic against all 4 serotypes in flavivirus-naive adults. J. Infect. Dis. 201 370-377 (2010)

104. Guy, B. et al. Cell-mediated immunity induced by chimeric tetravalent dengue vaccine in naive or flavivirus-primed subjects. Vaccine 26, 5712-5721 (2008).

105. Blaney, J. E. Jr, Durbin, A. P., Murphy, B. R. \& Whitehead, S. S. Development of a live attenuated dengue virus vaccine using reverse genetics. Viral Immunol. 19, 10-32 (2006).

106. Huang, C. Y. et al. Dengue 2 PDK-53 virus as a chimeric carrier for tetravalent dengue vaccine development. J. Virol. 77, 11436-11447 (2003).

107. Smucny, J. J., Kelly, E. P., Macarthy, P. O. \& King, A. D. Murine immunoglobulin $\mathrm{G}$ subclass responses following immunization with live dengue virus or a recombinant dengue envelope protein. Am. J. Trop. Med. Hyg. 53, 432-437 (1995)

108. Beaumier, C. M. et al. Differential in vivo clearance and response to secondary heterologous infections by $\mathrm{H} 2^{\mathrm{b}}$-restricted dengue virus-specific $\mathrm{CD} 8{ }^{+} \mathrm{T}$ cells. Viral Immunol. 23, 477-485 (2010).

109 Shresta, S et al. Interferon-dependent immunity is essential for resistance to primary dengue virus infection in mice, whereas T- and B-cell-dependent immunity are less critical. J. Virol. 78, 2701-2710 (2004)

110. Kaufman, B. M., Summers, P. L., Dubois, D. R. \& Eckels, K. H. Monoclonal antibodies against dengue 2 virus E-glycoprotein protect mice against lethal dengue infection. Am. J. Trop. Med. Hyg. 36, 427-434 (1987)

111. Chen, H. C., Hofman, F. M., Kung, J. T., Lin, Y. D. \& Wu-Hsieh, B. A. Both virus and tumor necrosis factor alpha are critical for endothelium damage in a mouse model of dengue virus-induced hemorrhage. J. Virol. 81, 5518-5526 (2007)

112. Souza, D. G. et al. Essential role of platelet-activating factor receptor in the pathogenesis of dengue virus infection. Proc. Natl Acad. Sci. USA 106 14138-14143 (2009)

113. Huang, K. J. et al. Manifestation of thrombocytopenia in dengue-2-virus-infected mice. J. Gen. Virol. 81 2177-2182 (2000).

114. Johnson, A. J. \& Roehrig, J. T. New mouse model for dengue virus vaccine testing. J. Virol. 73, 783-786 (1999).

115. Shresta, S., Sharar, K. L., Prigozhin, D. M., Beatty, P. R $\&$ Harris, E. Murine model for dengue virus-induced lethal disease with increased vascular permeability. J. Virol. 80, 10208-10217 (2006).

116. Koraka, P., Benton, S., van Amerongen, G., Stittelaar, K. J. \& Osterhaus, A. D. Efficacy of a live attenuated tetravalent candidate dengue vaccine in naive and previously infected cynomolgus macaques. Vaccine 25, 5409-5416 (2007).

117. Guirakhoo, F. et al. Safety and efficacy of chimeric yellow fever-dengue virus tetravalent vaccine formulations in nonhuman primates. J. Virol. 78 4761-4775 (2004)

Competing interests statement

The author declares competing financial interests: see Web version for details.

FURTHER INFORMATION Alan L. Rothman's homepage: http://www.immunome.org ALL LINKS ARE ACTIVE IN THE ONLINE PDF 